\title{
Controlled-Release oxygen Using polymers
}

\author{
Hongpo Wang, Hong Zhou,Hao Liu,Tong Yuan,Xuejiang Li \\ School of Computer Science and Information Engineering, Tianjin Agricultural \\ University,Tianjin,300384,China zhouhong@tjau.edu.cn
}

\begin{abstract}
Keywords: controlled-release; oxygen; polymers; Cytotoxicity
Abstract-- In tissue regenerative cell therapy is an important limitation is the initial hypoxia. In order to increase growth and cell survival after implantation of our oxygen release material were tested. Calcium peroxide polymer matrix used as the oxygen source. Two polymers to slow and prolonged release oxygen, L-lactic acid and lactic acid-co-glycolic acid test period. With respect to the calcium peroxide particles,Both delivery systems were initially higher,Shorter oxygen release. Human mesenchymal stem cells Cast films develop these oxygen-releasing composite material We need catalase proliferation, suggesting production Cytotoxicity hydrogen peroxide as intermediate. Catalase can be reduced through the cell cytotoxicity of hydrogen peroxide.
\end{abstract}

\section{Introduction}

Failure to replace the use of somatic cell therapy tissue at the implant site by a lack of vessel obstruction, leading to cell death from cell implantation. An important factor leading to cell death is believed to be the oxygen and nutrients and a limited number of cells to get rid of waste disability. One potential solution to the lack of oxygen can be delivered using the composite oxide scaffold, as an oxygen source having a peroxide salt. These materials should provide oxygen to the cells or tissues extended time period, such that implanted cell survival and to promote tissue repair, but not angiogenesis, which is causing the interference hypoxic conditions. This composite material should be available throughout the stent cell survival, thus improving tissue restoration or repair. Oxygen release should continue until the angiogenic process is complete and a new generation of functional vascular network. By reaction with the peroxide to provide oxygen in water (Formula 1). In the reaction intermediates, hydrogen peroxide is considered to be a cytotoxic agent. Mammalian cells have some defensive mechanisms to help convert hydrogen peroxide and water, and can be treated with low concentrations of hydrogen peroxide.

$$
\begin{aligned}
& \mathrm{CaO} 2+2 \mathrm{H} 2 \mathrm{O}=\mathrm{Ca}(\mathrm{OH}) 2+\mathrm{H} 2 \mathrm{O} 2 \\
& 2 \mathrm{H} 2 \mathrm{O} 2=2 \mathrm{H} 2 \mathrm{O}+\mathrm{O} 2
\end{aligned}
$$

Oxygen delivery, a careful balance between angiogenesis and cell toxicity is required. Oxygen release in this study A composite material, which consists of calcium peroxide powder Embedded in a biodegradable poly (lactic acid) (PLA) and Poly (lactic - co - glycolic acid) (PLGA), were evaluated Their oxygen delivery capacity and their cytotoxicity Human bone marrow mesenchymal stem cells. Polymer matrix is intended to serve as a barrier to both the inflow and outflow of water and an active agent, slowing down the reaction and reduce cytotoxicity. Therefore, we assume that PLA and PLGA polymers obstacle extended release oxygen, reduce cytotoxicity. Due to the fast degradation of PLGA than PLA, PLGA-based composite is expected to show a faster release of oxygen.

\section{Materials and methods}

Solutions of DL-lactide-co-glycolide and DL-Lactide in chloroform were prepared at ten percent. Five percent of the calcium peroxide powder was dispersed and stirred vigorously. Oxygen 
measuring, the suspension was pipetted $1 \mathrm{~mL}$ in $50 \mathrm{~mL}$ vial, and dried at room temperature for 48 hours, and 24 hours in vacuum. Measure oxygen delivery is at 37 degrees in $35 \mathrm{ml}$ deoxygenated SBF oxygen instrument by WTW OXI3310. Oxygen measuring device is in an open anaerobic system through continuous input and out of $\mathrm{N}_{2}$ gas. Calcium peroxide from oxygen separately from the release of $5 \mathrm{mg}$ of calcium peroxide is filled in the porous filter paper placed in a flask, was then charged with a base of assessment $35 \mathrm{ml}$ SBF.

For cell culture $15 \mathrm{~mm}$ cover glasses are coated $2501 \mu \mathrm{L}$ suspended and dried as described above. It does not contain calcium peroxide, control material produced by a $10 \%$ polymer solution. Isolated from a patient receiving a total hip arthroplasty bone marrow, hMSCs were cultured in $\alpha$-MEM supplemented with $10 \%$ heat-inactivated fetal bovine serum, $0.2 \mathrm{mM}$ ascorbic acid - acid 2-phosphate and $1 \%$ antibacterial - antifungal solution in the wet atmosphere supplemented with $5 \% \mathrm{CO} 2$, and treated with trypsin -EDTA solution at 70-80\% confluence. To assess the cytotoxic effect in containing PLA, PLA 24-well plates/Calcium peroxide, PLGA, PLGA / calcium peroxide-coated cover glass square The hMSCs / well, and cultured in normoxic or hypoxic conditions at $37 \mathrm{C}, 5 \% \mathrm{CO}_{2}$ and $100 \%$ humidity 2 and 5 days. It should be noted that the catalase was added to the cell culture in a concentration of $100 \mathrm{U} / \mathrm{ml}$. Be evaluated by the XTT Cytotoxicity Analysis. The absorbance at 460 and $690 \mathrm{~nm}$ read using a microplate reader Fluostar ship. Morphological examinations were destroyed non-transparent and high background fluorescence cytochemistry made of composite material, probably due to the presence of peroxides. It is not expected to alter cell differentiation occurs in the incubation period of five days. Statistical analysis was performed using one-way ANOVA, and post hoc test of SPSS. Time variables are evaluated in the presence of material and catalase enzyme.

\section{Results and discussion}

Composites and calcium peroxide releases oxygen particles identified in an open system, flushed with nitrogen at $37{ }^{\circ} \mathrm{C}$. Calcium peroxide in the PLA and PLGA embedded lead to a faster release of oxygen compared to the release of a single from the calcium peroxide particles (Fig. 1). The amount of the polydimethylsiloxane embedded calcium peroxide crystals to reduce water inflow and outflow of hydrogen peroxide, resulting in a very efficient oxygen delivery system. However, PDMS is a non-degradable material and has been shown to have small-cell adhesion. Hydrolysis of lactide based polymers may lead to reduced $\mathrm{pH}$ value, thereby inducing the intermediate $\mathrm{Ca}(\mathrm{OH}) 2$ higher solubility of the shift reaction to produce hydrogen peroxide. Oxygen release PLGA-based composite materials, previously used Harrison and oh, can be combined with $\mathrm{Na}_{2} \mathrm{CO}_{3} \sim 1.5 \mathrm{H}_{2} \mathrm{O}_{2}$ or calcium peroxide. Release kinetics of oxygen found in these studies are difficult to compare our data, because a closed system these researchers used to assess oxygen release and our setup is an open system is more similar to in vivo conditions. Furthermore, different manufacturing methods provide oxygen compound compared to our in their material, which may have reduced the water inflow.

The overall evaluation of our open system settings and does not allow oxygen from material sent. MSC grown in PLGA or PLA surface showed better viability than hMSC growth in calcium peroxide (Fig. 2). hMSC grown on PLA/CaO2 constructs exhibited XTT values of $0.508(\mathrm{t}=1)$ and $0.877(\mathrm{t}=7)$, whereas the XTT conversion by hMSC on PLGA/CaO2 constructs revealed values of $0.244(\mathrm{t}=1)$ and $0.91(\mathrm{t}=7)$. If the number of TXT conversion and related cells, then the cells in PLGA PLA/calcium peroxide / calcium peroxide grew faster it. HMSC initial adhesion seems to be PLA/calcium peroxide better. Oxygen from the Special PLGA / calcium peroxide burst can cause this reduced cell viability at $\mathrm{t}=1$. We had a relatively high concentration of hydrogen peroxide cells 
survived and grown in PLGA/calcium peroxide surface, and then better in the PLA/calcium peroxide surface anoxic conditions. The ability of catalase significantly improved conversion TXT of hMSC. On cells PLGA/calcium peroxide cultured XTT value but varies between 0.127 and 0.253 catalase, and catalase values were 0.24 and 0.91 . hMSCs and is not significantly affected by the choice of anoxic conditions, whether it is grown in the polymer structure or tissue culture polystyrene (TCPS), XTT conversion even appeared to increase after 7 days. In a long culture period on $0.1 \%$ of oxygen hMSC TCPS and polymer surface does exhibit reduced viability, described by reducing the number of cells. sMSC cultured in medium supplemented with additional glucose. These researchers alter blood glucose concentration in the medium, but not in 12 days of hypoxia (1\%) to refresh the media during culture. Nevertheless, sMSC showed almost no signs of cell death. A similar effect is observed using the normal concentration of glucose in our study, but refresh the media twice a week. Lack of glucose resulting in a closed culture system may not be achieved.

Companies registered in the PLA or PLGA calcium peroxide oxygen did not result in a slow-release system. The most likely reason is that the acidity of the degradation products of the polymer. Intermediate reaction product $\mathrm{Ca}(\mathrm{OH}) 2$ may stimulate the hydrolysis of the polymer by increasing the $\mathrm{pH}$ value. Using PLA and PLGA has a limited applicability in slow oxygen release system.

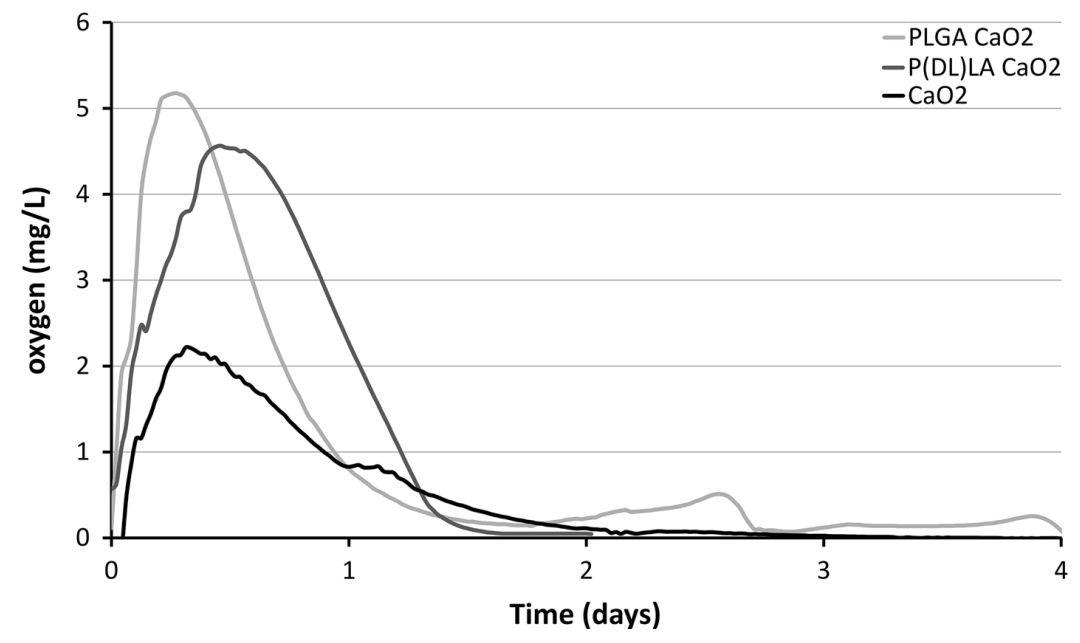

Fig. 1 Oxygen release in time
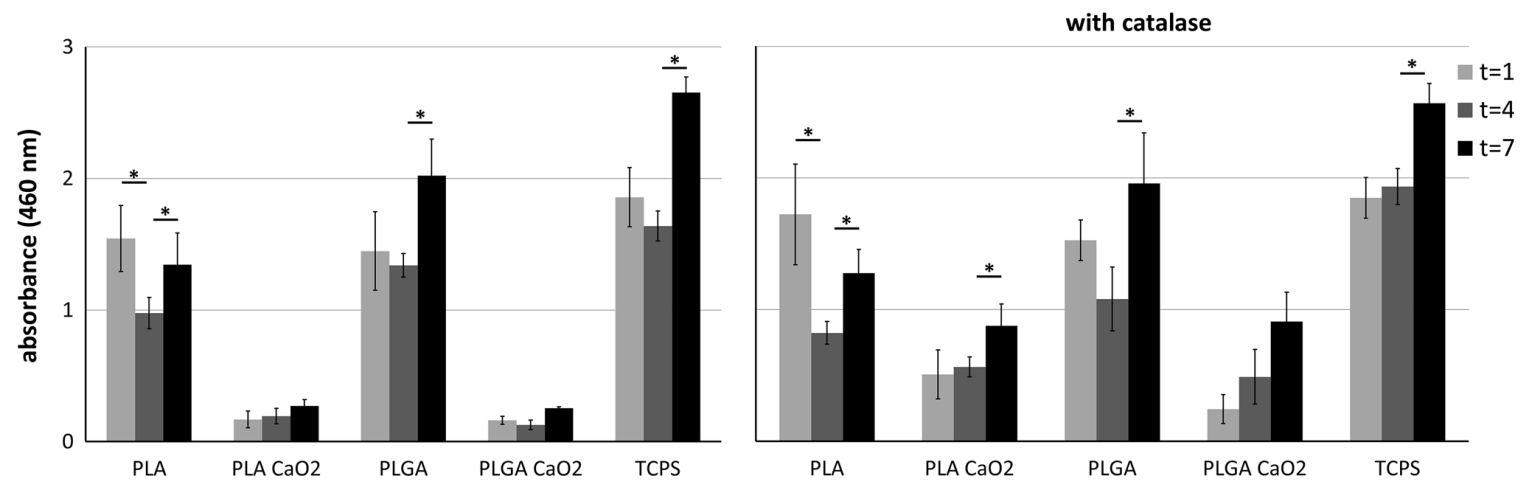

Fig. 2 Histogram representing absorbance values of the XTT viability assay.

\section{Conclusion}

Prepared by embedding embedding either PLA or PLGA polymer particles of calcium peroxide biodegradable, releasing oxygen composites exhibit enhanced oxygen release compared to free calcium peroxide. Polymer - peroxide construct seems moderate cytotoxicity. Catalase can be 
reduced cytotoxicity, suggesting that the role of $\mathrm{H}_{2} \mathrm{O}_{2}$. PLA and PLGA polymers suitable for sustained release less oxygen system.

\section{Acknowledgements}

The authors gratefully acknowledge financial support from the national spark plan(10ZHXHNC06700),School Youth Doctor Fund(2012D05)and School Teaching Reform fund(12-A2-05)

\section{References}

[1] Colton CK. Oxygen supply to encapsulated therapeutic cells. Adv Drug Deliv Rev. 2014:93-110.

[2] Pedraza E, Coronel MM, Fraker CA, Ricordi C, Stabler CL.Preventing hypoxia-induced cell death in beta cells and islets via hydrolytically activated, oxygen-generating biomaterials. Proc Natl Acad Sci USA. 109(42),2012:45-50.

[3] L. Li, H. Li, Y. Qian, et al., Electrospun poly (varepsilon-caprolactone)/silk fibroin core-sheath nanofibers and their potential applications in tissue engineering and drug release, Int. J. Biol. Macromol. 49 (2) 2011:223-232.

[4] C.N. Manning, H.M. Kim, S. Sakiyama-elbert, L.M. Galatz, N. Havlioglu, S. Thomopoulos, Sustained delivery of transforming growth factor beta three enhances tendon-to-bone healing in a rat model, J. Orthop. Res. 2011:1099-1105.

[5] Q. Jin, P.X. Ma, W.V. Giannobile, Platelet-derived growth factor delivery via nanofibrous scaffolds for soft-tissue repair, Adv. Skin Wound Care 1,2010:375-381.

[6] N.A. Impellitteri, M.W. Toepke, S.K. Lan Levengood, W.L. Murphy, Specific VEGF sequestering and release using peptide-functionalized hydrogel microspheres, Biomaterials 33 (12), 2012:3475-3484.

[7] Cruse, M. J.; Liebman, M.; Raman, D. R.; Wiedenhoeft, M. H. Fossil energy use in conventional and low-external input cropping systems. Agron. J. 102,2010: 934-941.

[8] Nie, X.; Besant, R. W.; Evitts, R. W. An experimental study of moisture uptake and transport in a bed of urea particles. Granul. Matter 10,2008:301-308. 